\title{
Foveolar gastric metaplasia presenting as a duodenal tumor with an atypical appearance: a case report
}

\author{
Takahiro Abe ${ }^{1}$, Masayuki Kato ${ }^{1 *}$, Shuzo Kohno², Sigeharu Hamatani ${ }^{3}$, Yosuke Kawahara ${ }^{1}$, Kimio Isshi $^{1}$, \\ Koji Matsuda ${ }^{1}$ and Kazuki Sumiyama ${ }^{4}$
}

\begin{abstract}
Background: Foveolar gastric metaplasia of the duodenum is a frequent but not as yet considered correlate of endoscopically detected duodenal polyps. The majority of foveolar gastric metaplasias associated with polyps presented a typical benign endoscopic appearance and they were diagnosed by biopsy. Here we report a case of a surgical-resected foveolar gastric metaplasia manifesting as a duodenal tumor with an atypical appearance.

Case presentation: An asymptomatic 56-year-old Asian man who presented with a foveolar gastric metaplasia of atypical appearance and had previously undergone esophagogastroduodenoscopy was referred to our hospital. A biopsy revealed a normal duodenum with an inflamed mucosa. Narrow band imaging with magnifying endoscopy revealed normal microvessels with normal micromucosa, which indicated non-neoplasia. Endoscopic ultrasonography using a miniature probe system $(20 \mathrm{MHz})$ revealed a hypoechoic mass with multiple anechoic lesions (16-mm diameter) located in the mucosal layer. The lesion was excised via laparotomy assisted by endoscopic techniques similar to endoscopic submucosal dissection. The pathology indicated foveolar gastric metaplasia.

Conclusions: Foveolar gastric metaplasia can present as a duodenal tumor. We identified two important clinical issues. First, foveolar gastric metaplasia can present as a duodenal tumor with an atypical benign appearance. Second, both endoscopic ultrasonography and narrow band imaging are useful techniques to increase the diagnostic rate of this condition.
\end{abstract}

Keywords: Foveolar gastric metaplasia (FGM), Ectopic gastric mucosa, Narrow band imaging (NBI), Endoscopic ultrasonography (EUS)

\section{Background}

Foveolar gastric metaplasia (FGM) of the duodenum is a frequent but not as yet considered correlate of endoscopically detected duodenal polyps. According to a comparison of findings from two institutes with upwards of 100,000 gastroenterological cases per year, the association of FGM with typical polyp morphology was shown to be at higher rates than previously identified [1]. Results from this comparison showed a $41 \%$ association in one institute and $32 \%$ in the other institute. The

\footnotetext{
* Correspondence: masakato89@gmail.com

${ }^{1}$ Department of Endoscopy, The Jikei University Katsushika Medical Center,

6-41-2 Aoto Katsushika-ku, Tokyo 125-8506, Japan

Full list of author information is available at the end of the article
}

majority of cases of FGM associated with polyps presented a typical benign endoscopic appearance and they were diagnosed by biopsy. Here we report a case of a surgical-resected FGM manifesting as a duodenal tumor with an atypical appearance.

\section{Case presentation}

An asymptomatic 56-year-old Asian man who presented with an FGM of atypical appearance and had previously undergone esophagogastroduodenoscopy was referred to our hospital. He had taken antihypertensive medicine for hypertension. He denied alcohol intake and did not smoke tobacco. An abdominal observation revealed that his abdomen was soft and flat and without pain. His 
neurological findings were normal. The results of laboratory findings were normal (Table 1). A biopsy revealed a normal duodenum with an inflamed mucosa. The tissue proximal to the esophagogastroduodenoscopy showed a wide-based sessile submucosal tumor-like mass. A 2-cm depression on top of the lesion was observed in the anterior wall of the second part (descending) of his duodenum. A biopsy was performed and repeated, with both results showing no malignancy. Narrow band imaging (NBI) with magnifying endoscopy revealed normal microvessels with normal micromucosa, which indicated non-neoplasia. Endoscopic ultrasonography (EUS) using a miniature probe system $(20 \mathrm{MHz})$ revealed a hypoechoic mass with multiple anechoic lesions (16-mm diameter) located in the mucosal layer (Fig. 1). Although the evidence suggested that the lesion was benign, other causes including lymphoma, ectopic pancreatic tissue, carcinoid, or leiomyosarcoma of the duodenum were considered. Therefore, the lesion was excised via laparotomy assisted by endoscopic techniques, similar to endoscopic submucosal dissection (ESD). The pathology indicated FGM without gastric glands (Fig. 2). He was discharged from our hospital 13 days after surgery without postoperative complications.

The esophagogastroduodenoscopy showed no recurrence of the lesion after 10 months.

\section{Discussion}

We identified two important clinical issues: FGM can present as a duodenal tumor with an atypical benign appearance, and both EUS and NBI are useful techniques to increase the diagnostic rate of this condition.

First, FGM can present as a duodenal neoplasia with an atypical benign appearance. FGM is common in the duodenum, with localized ectopic gastric mucosa [2]. A search of the PubMed and Medline databases, at the

Table 1 The result of laboratory findings

\begin{tabular}{ll}
\hline White blood cell count $(\mu \mathrm{l})$ & 8300 \\
Red blood cell count $\left(10^{3} / \mu \mathrm{l}\right)$ & 506 \\
Hemoglobin $(\mathrm{g} / \mathrm{dl})$ & 15.7 \\
Aspartate transaminase $(\mathrm{U} / \mathrm{L})$ & 20 \\
Alanine transaminase $(\mathrm{U} / \mathrm{L})$ & 29 \\
Alkaline phosphatase $(\mathrm{U} / \mathrm{L})$ & 157 \\
Total protein $(\mathrm{g} / \mathrm{dL})$ & 7.2 \\
Creatinine $(\mathrm{mg} / \mathrm{dl})$ & 0.66 \\
Sodium $(\mathrm{Na} ; \mathrm{mmol} / \mathrm{L})$ & 138 \\
Potassium $(\mathrm{K} ; \mathrm{mmol} / \mathrm{L})$ & 4.6 \\
C-reactive protein $(\mathrm{mg} / \mathrm{dl})$ & 0.1 \\
Carcinoembryonic antigen $(\mathrm{CEA} ; \mathrm{ng} / \mathrm{ml})$ & 4.3 \\
Cancer antigen $(\mathrm{CA} 19-9 ; \mathrm{U} / \mathrm{ml})$ & 7 \\
\hline
\end{tabular}

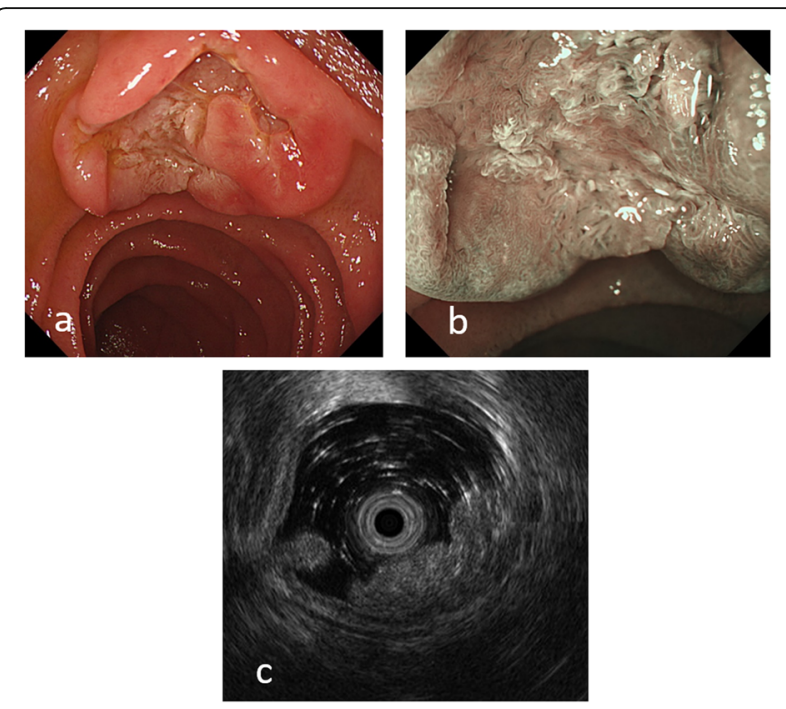

Fig. 1 a An endoscopic picture showing a wide-based sessile submucosal tumor-like mass with depression at the top of the lesion. b Narrow banding image with magnification showing non-irregular microvessel or mucosal structure. c Endoscopic ultrasound images showing a heterogeneously hyperechoic mass with several anechoic lesions located predominantly in the mucosa

Jikei University Library of Medicine, using the keyword "ectopic (heterotopic) gastric mucosa duodenum" or "foveolar gastric metaplasia duodenum" identified two reports that indicated that resections were performed to rule out malignancies in ectopic gastric mucosa with an abnormal appearance $[3,4]$. With particular regard to FGM, the present case study is the first to differentiate between malignant and benign cases. For this case, a decision was made to resect the lesion via endoscopyassisted laparotomy. After making circumferential endoscopic incisions, similar to a technique used in ESD, the lesion was surgically excised via laparotomy.

Second, both EUS and NBI were useful to increase the diagnostic rate of FGM. According to the validity report for EUS for ectopic gastric mucosa lesions, endoscopic resection may be used in cases of duodenal ectopic gastric mucosa when EUS can verify that the tumor is localized in the third layer, which corresponds to the submucosa of the duodenal wall [3]. In this case, EUS results verified that the lesion was located in the mucosal layer and was demonstrated to be a heterogeneously hypoechoic mass with small anechoic areas (Fig. 1). A few reports have described the diagnostic value of NBI in duodenal endoscopy [5]. In the present case, using $\mathrm{NBI}$, we ruled out duodenal tumor because of the absence of irregular microvessel and micromucosal patterns. Multiple biopsy results supported the NBI findings that the lesion was not malignant.

In our case, although the lesion was diagnosed as benign, surgical resection was scheduled because of the 


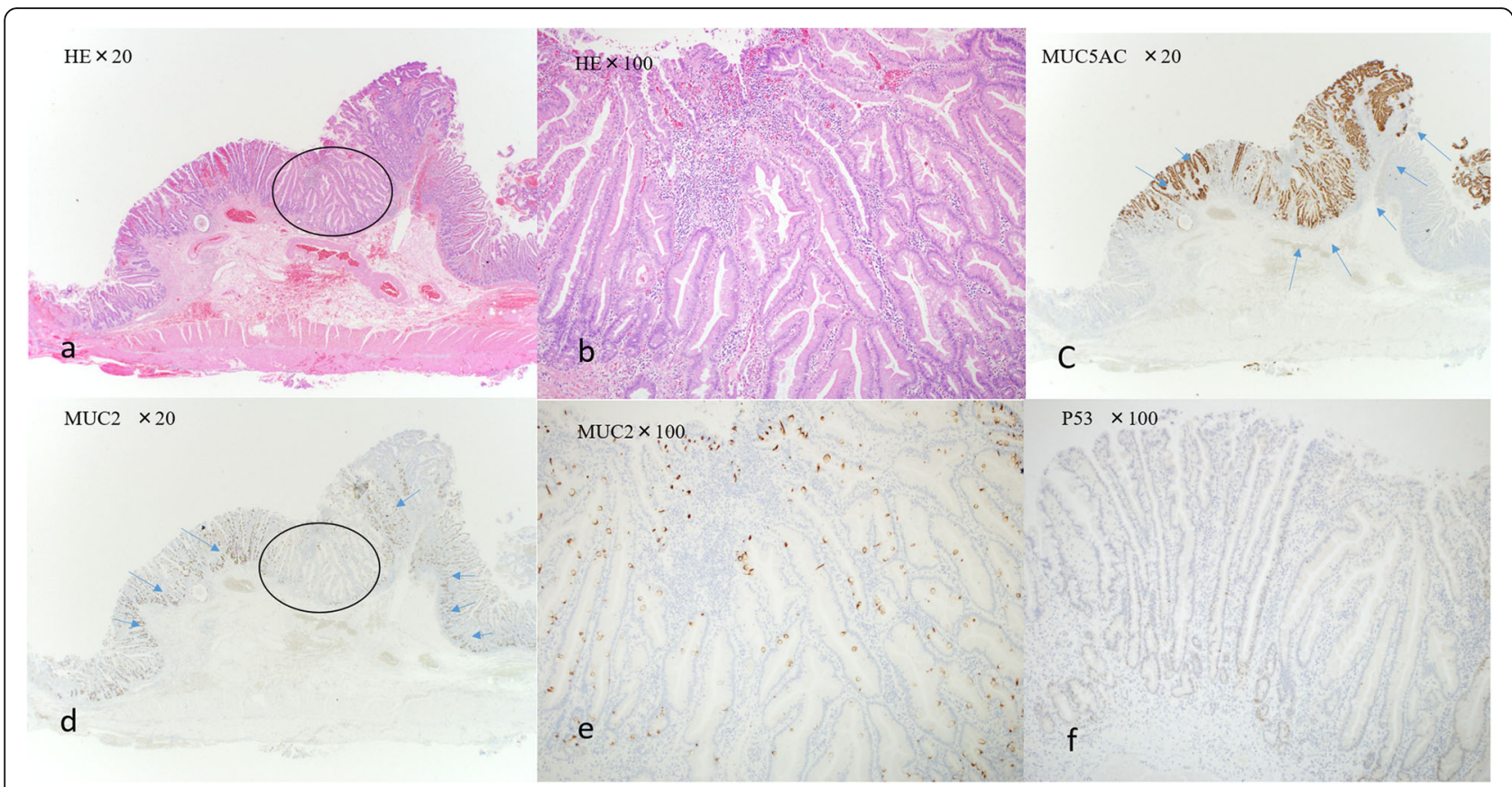

Fig. 2 a Photomicrograph of the resected duodenal specimen shows irregular sequence of the tubular epithelium; hematoxylin and eosin (H\&E), original magnification, $\times 20$. b Hematoxylin and eosin (original magnification, $\times 100$ ). $\mathbf{c}$ Immunohistological staining of foveolar epithelium for mucin 5AC (MUC5AC). No staining of the original duodenal epithelium. Brown cytoplasmic staining of the foveolar gastric metaplasia (blue arrows; magnification, $\times 20$ ). $\mathbf{d}$ Immunohistological staining of goblet cell in duodenum mucin 2 (MUC2). No staining of the lesion. Brown staining of the original duodenal epithelium (magnification, $\times 20$ ). e Mucin 2 (magnification, $\times 100$ ). $\mathbf{f}$ Immunohistological staining of a malignant potential for p53. No staining of p53 (magnification, $\times 100)$. The circled within the panel denotes high power of H \& E stained slide $(a \rightarrow b)$ and MUC2 $(d \rightarrow e)$

relatively large size and unusual morphology of the lesion. We must concede that because the lesion was identified as benign, an observation period could have been an acceptable alternative. However, multiple factors led us to proceed with our chosen course of action, and valuable information was obtained.

\section{Conclusions}

FGM can present as a duodenal tumor for which both EUS and NBI are valuable diagnostic tools. FGM can present as a duodenal tumor with an atypical benign appearance, and this should be considered while treating patients in future. In fact, many occurrences may remain unrecognized and undetected. EUS and NBI are highly recommended to verify and diagnose FGM before resection is considered. Further reporting would be useful to support the evidence that the incidence of FGM may be higher than previously thought, resulting in undiagnosed and untreated cases.

\section{Abbreviations}

ESD: Endoscopic submucosal dissection; EUS: Endoscopic ultrasonography; FGM: Foveolar gastric metaplasia; NBI: Narrow band imaging

\section{Acknowledgements}

The authors have no disclosures to make and have had no source of funding in the preparation of this manuscript. We would like to thank $\mathrm{Mr}$ Wayne Thibaudeau for his support.

\section{Funding}

The authors declare that they have no funding.

\section{Availability of data and materials}

Not applicable.

\section{Authors' contributions}

TA was a major contributor to the writing of the manuscript. MK reviewed and was a major contributor to the writing of the manuscript. Endoscopic and surgical treatments were performed by MK and SK. SH performed the histological examination. $\mathrm{YK}, \mathrm{Kl}$, and $\mathrm{KM}$ contributed to the entire research. KS reviewed and approved the final version of the manuscript. All authors read and approved the final manuscript.

\section{Competing interests}

The authors declare that they have no competing interests.

\section{Consent for publication}

Written informed consent was obtained from the patient for publication of this case report and accompanying images. A copy of the written consent is available for review by the Editor-in-Chief of this journal.

\section{Ethics approval and consent to participate}

Not applicable.

\section{Author details}

'Department of Endoscopy, The Jikei University Katsushika Medical Center, 6-41-2 Aoto Katsushika-ku, Tokyo 125-8506, Japan. ${ }^{2}$ Department of Surgery, The Jikei University Katsushika Medical Center, 6-41-2 Aoto Katsushika-ku, Tokyo 125-8506, Japan. ${ }^{3}$ Department of Pathology, Clinical Service, The Jikei University Katsushika Medical Center, 6-41-2 Aoto Katsushika-ku, Tokyo 125-8506, Japan. ${ }^{4}$ Department of Endoscopy, The Jikei University School of Medicine, 3-25-8 Nishishinbashi Minato-ku, Tokyo 105-8461, Japan. 
Received: 3 September 2016 Accepted: 30 November 2016

Published online: 20 December 2016

\section{References}

1. Sarbia M, Sauer G, Karimi D, Berndt R. Foveolar gastric metaplasia of the duodenum: a frequent, so far neglected type of duodenal polyp. Z Gastroenterol. 2014;52(4):348-50.

2. Terada T. Heterotopic gastric mucosa of the gastrointestinal tract: a histopathologic study of 158 cases. Pathol Res Pract. 2011;207(3):148-50.

3. Eguchi K, Aoyagi K, Nimura S, Sakisaka S. Diagnostic value of endoscopic and endoscopic ultrasound characteristics of duodenal submucosal tumourlike heterotopic gastric mucosa. Can J Gastroenterol. 2011;25(7):365-7.

4. Shiraishi N, Yasuda K, Bandoh T, Adachi Y, Kaketani K, Kitano S. Laparoscopic resection for ectopic gastric mucosa of the duodenum: report of a case. Surg Today. 1999;29(4):351-3.

5. Kikuchi D, Hoteya S, lizuka T, Kimura R, Kaise M. Diagnostic algorithm of magnifying endoscopy with narrow band imaging for superficial nonampullary duodenal epithelial tumors. Dig Endosc. 2014;26(2):16-22.

Submit your next manuscript to BioMed Central and we will help you at every step:

- We accept pre-submission inquiries

- Our selector tool helps you to find the most relevant journal

- We provide round the clock customer support

- Convenient online submission

- Thorough peer review

- Inclusion in PubMed and all major indexing services

- Maximum visibility for your research

Submit your manuscript at www.biomedcentral.com/submit
Biomed Central 